\title{
Some of the recent topics on stochastic analysis
}

\author{
Takeyuki Hida $^{1}$ \\ Meijo University
}

Summary. First, we shall quickly explain why and how the space of generalized white noise functionals has been introduced. The space has big advantages to carry on the analysis of nonlinear functionals of white noise (or of Brownian motion) and to apply the theory to various fields. It should be noted that the introduction of generalized functionals was motivated by the Ito formula for Brownian functionals. Using this space we discuss the following two topics.

1. Path integrals. To formulate Lagrangian path integrals, we have to concretize the expressions of the Lagrangian in terms of paths. We propose that quantum mechanical paths (trajectories) are expressed as a sum of the classical paths and fluctuation which is taken to be a Brownian bridge. It is possible to give a plausible reason why a Brownian bridge is fitting in this case. With this choice of possible trajectories, there arises a difficulty that the kinetic energy becomes a generalized functional of a Brownian motion. It is now possible to overcome this difficulty to take our favorable space of generalized white noise functionals. Then follows the integration. Our method can be applied to a wider class of dynamics, for instance, to those cases with singular potentials and to some fields over non-euclidean space.

2. Infinite dimensional rotation group and unitary group.

It is well known that the "infinite dimensional rotation group" has naturally been introduced in connection with white noise, and the group describes certain invariance of the white noise measure. Hence, we may say that the white noise analysis should have an aspect of an infinite dimensional harmonic analysis. It seems natural, in fact by many reasons, to complexify the rotation group to have "infinite dimensional unitary group". Thus complexified group has various interesting applications to the analysis of nonlinear functionals of complex white noise. In addition, we can find good connections with Lie group theory and theory of quantum dynamics, to which we can give new interpretations.

\section{Introduction}

We are interested in essentially infinite dimensional analysis and discuss functionals of the form 


$$
f(B(s), s \in T, t) \rightarrow \varphi(\dot{B}(s), s \in T, t),
$$

where $\{\dot{B}(t)\}$ is a white noise and is a system of idealized elemental random variables.

\section{Flow Diagram}

1. Start with the Itô formula, the simplest case:

$$
(d B(t))^{2}=d t .
$$

Magnify $(d B(t))^{2}-E\left[\left(d B(t)^{2}\right]\right.$ (multiply by $\frac{1}{(d t)^{2}}$ to have $\left.: \dot{B}(t)^{2}:\right)$.

Generally, renormalized monomials in $\dot{B}(t)$ 's.

2. Space of generalized white noise functionals by using

i) Wiener-Itô Decomposition of $\left(L^{2}\right)$,

ii) $S$-transform.

\section{Analysis}

Harmonic Analytsis arising from rotation group.

\section{Two proposed directions}

i) Path integrals,

ii) Infinite dimensional unitary group.

\section{Our idea of white noise analysis}

The idea to discuss random complex systems is based on the "Reductionism". Actual implementation is to construct the innovation by extracting necessary and sufficient information from the given random complex system. This is the first step of our mathematical approach to the study of random complex systems. The standard innovation can be obtained as the time derivative of a Lévy process. We are thus given an elemental random system. This choice is quite reasonable for our purpose. 
Then, follows the next step "Synthesis". There the given random complex system should be expressed as a functional (which is non-random and, in general, nonlinear functional) of the innovation that has just been obtained in the step of the reduction. Thus, we have an analytic representation of the random complex phenomenon in question by using functions known in functional analysis.

Finally, we are ready to study the "Analysis" of the functionals, in fact, nonlinear functionals of the innovation. It can be proceeded having been suggested by the ordinary functional analysis. Further various applications can be discussed, and even one can see beautiful interplay between our theory and the studies of actual problems in various fields of science.

To be added, there are interesting applications in various fields of science, some of which will be presented in the present notes.

We are sure that the innovation approach is one of the most efficient and legitimate directions to the study of stochastic processes and random fields, or more generally to random complex systems. See [7].

We now focus our attention on the case, where the innovation is Gaussian.

\section{Generalized white noise functionals.}

Having had the system of variables to be $\{\dot{B}(t)\}$, we are naturally led to introduce basic functions of the $\dot{B}(t)$ 's. We had a naive observation on the square of $\dot{B}(t)$. A particular case of the Itô formula $(d B(t))^{2}=d t$ derives

renormalized variable : $\dot{B}(t)^{2}$ :. It makes sense as a generalized functional of white noise.

Remark L. Accardi has done profound research on powers of $\dot{B}(t)$ 's.

With these facts in mind we define classes of generalized white noise functionals, which are the most important concepts in white noise theory.

Our starting point is the Fock space for $\left(L^{2}\right)$, that involves ordinary white noise functionals with finite variance,

$$
\left(L^{2}\right)=\sum_{0}^{\infty} \bigoplus H_{n},
$$

where $H_{n}$ is the space of multiple Wiener integrals in Itô sense of degree $n$.

There are two typical ways of introducing generalized white noise functionals.

I. Use of the Sobolev spaces $K^{m}\left(R^{n}\right)$ over $R^{n}$ of degree $m$. 


$$
\widehat{K^{m}\left(R^{n}\right)} \subset \widehat{L^{2}\left(R^{n}\right)} \subset \widehat{K^{-m}\left(R^{n}\right)}
$$

where the both inclusions are continuous injections.

Our favorable choice of the degree $m$ of the Sobolev space is $(n+1) / 2$. For one thing, members in $K^{(n+1) / 2}\left(R^{n}\right)$ are continuous and the restriction of them to an $(n-1)$-dimensional hyperplane belongs to the space $\left.K^{n / 2\left(R^{n}-1\right.}\right)$, namely the degree decreases by $1 / 2$ when the restriction is made to a subspace of one dimension lower.

Let $m=(n+1) / 2$ in the above triple involving symmetric Sobolev spaces. We have

$$
K^{(n+1) / 2}\left(R^{n}\right) \subset \widehat{L^{2}\left(R^{n}\right)} \subset K^{-(\widehat{n+1) / 2}}\left(R^{n}\right),
$$

and we form

$$
H_{n}^{(n)} \subset H_{n} \subset H_{n}^{(-n)},
$$

each space of this triple is isomorphic to the corresponding symmetric Sobolev space. The norms in those spaces are denoted by $\|\cdot\|_{n},\|\cdot\|$, and $\|\cdot\|_{-n}$. Thus, we have a Hilbert space $H_{n}^{(-n)}$ of generalized white noise functionals of degree $n$.

There remains a freedom on how to sum up the spaces $H_{n}^{(-n)}, n \geq 0$. Choose an increasing sequence $c_{n}>0$, and form a Hilbert space $\left(L^{2}\right)^{+}$by the direct sum

$$
\left(L^{2}\right)^{+}=\bigoplus c_{n} H_{n}^{(n)}
$$

The direct sum forms a Hilbert space and its dual space is expressed in the form

$$
\left(L^{2}\right)^{-}=\bigoplus c_{n}^{-1} H_{n}^{(-n)}
$$

Naturally, we are given a triple

$$
\left(L^{2}\right)^{+} \subset\left(L^{2}\right) \subset\left(L^{2}\right)^{-}
$$

The space $\left(L^{2}\right)^{+}$consists of test functionals, while $\left(L^{2}\right)^{-}$is the space of generalized white noise functionals (For details, see [8], Chap.2).

II. An analogue of the Schwartz space.

Take the parameter space $R$ and an operator $A$ :

$$
A=-\frac{d^{2}}{d u^{2}}+u^{2}+1
$$


acting on $L^{2}(R)$. Then, apply the second quantization technique to introduce the operator $\Gamma(A)$ acting on the space $\oplus \widehat{L^{2}\left(R^{n}\right)}$. By using the isomorphism $\pi$ defined in I:

$$
\pi: H_{n} \longrightarrow \widehat{L^{2}\left(R^{n}\right)}
$$

we can easily define the operator $\tilde{\Gamma}(A)=\pi^{-1} \Gamma(A) \pi$. It is proved, as in I, that for $\varphi \in H_{n}$

$$
\|\varphi\|=\sqrt{n !}\|\pi \varphi\|_{L^{2}\left(R^{n}\right)} .
$$

Unless no confusion occurs, $\tilde{\Gamma}(A)$ is also denoted by $\Gamma(A)$. It acts on $\left(L^{2}\right)$.

Set $(S)_{n}=\mathcal{D}\left(\Gamma(A)^{n}\right)$ and set

$$
(S)=\bigcap_{n}(S)_{n} .
$$

The projective limit topology is introduced to $(S)$.

The dual space of $(S)_{n}$ is denoted by $(S)_{-n}$ and its inductive limit:

$$
(S)^{*}=\lim (S)_{-n}
$$

is formed. The $(S)^{*}$ is the space of generalized white noise functionals. It is often called the space of white noise distributions.

Remind the $T$-transform for $\varphi \in\left(L^{2}\right)$ :

$$
(T \varphi)(\xi)=\int_{E^{*}} \exp [i<x, \xi>] \varphi(x) d \mu(x),
$$

and the $S$-transform

$$
(S \varphi)(\xi)=C(\xi) \int_{E^{*}} \exp [<x, \xi>] \varphi(x) d \mu(x) .
$$

They give us visualized and convenient representations of generalized white noise functiuonals.

\section{White noise approach to path integrals}

Our method for path integrals in quantum dynamics is to take white noise measure to define average (or expectation) of functionals, and to take generalized functionals in the integrand to have visualized expression. The path 
integral method has been originated by R. Feynman, with some motivation due to Dirac's idea, and it is viewed as a third method of quantization different from those by W. Heisenberg and E. Schrödinger. Our method of path integrals follows mainly the Feynman's idea in methodology, however, some new techniques are introduced.

In quantum dynamics there are many possible paths (trajectories) of a particle, and each of them is viewed as a sum of the classical trajectory and fluctuation. We assert that the amount of the fluctuation is expressed as a Brownian bridge.

First, we need to give a characterization of a Brownian bridge $X(t), t \in$ $[0,1]$, over the unit time interval, since it plays a key role in our setup. A Brownian bridge is a Gaussian Markov process with mean 0 and covariance function $E(X(t) X(s))=\Gamma(t, s)=(t \wedge s)(1-t \vee s), s, t \in[0,1]$.

Heuristically speaking, it was 1981 when we proposed a white noise approach to path integrals to have quantum mechanical propagators (see [13] appeared later in 1983). We had, at that time, some idea in mind for the use of a Brownian bridge and had many good examples that have quite wider class of potentials, and we obtained various satisfactory results.

Now it is time to recall the original idea by characterizing the Brownian bridge taking some physical intuition (see e.g. [13]) into account, so that we can explain why a Brownian bridge is fitting for describing the fluctuation around the classical path. We now have a theorem :

Theorem The Brownian bridge $X(t)$ over the interval $[0,1]$ is charcaterized (up to constant) by the conditions

i) $X(t)$ is a Gaussian Markov process that has the canonical representation,

ii) $X(0)=X(1)=0$ (bridged), and $E(X(t)) \equiv 0$,

iii) the normalized process $Y(t)$ enjoys the projective invariance,

iv) the local continuity of $Y(t)$ as $t \rightarrow 0$ in terms of covariance function is the same as that of the normalized Brownian motion $B(t) / \sqrt{t}$.

Proof. Assumptions i) - iii) proves that the covariance function $\Gamma(t, s)$ of the process to be determined has to be of the form

$$
\Gamma(t, s)=f\left(\frac{s}{1-s}\right) / f\left(\frac{t}{1-t}\right) .
$$

The assumption iv) asserts that $f$ is the square root of a variable. Thus, the theorem is proved. 
The Brownian bridge determined above has a canonical representation that is expressed in the form (up to constant):

$$
X(t)=(1-t) \int_{0}^{t} \frac{1}{(1-u)} \dot{B}(u) d u .
$$

The covariance function $\Gamma(t, s)$ of the normalized process $Y(t)=X(t) /$ $\sqrt{E\left(X(t)^{2}\right)}$ is of the form

$$
\Gamma(t, s)=\sqrt{(0,1 ; s, t)},
$$

where $(0,1 ; s, t)$ denotes the anharmonic ratio, that is $\frac{(1-t) / t}{(1-s) / s}$.

By observing the expression of the covariance function, it is obvious that a Brownian bridge is reversible in time, and further the theorem implies, as was announced before, that the Brownian bridge is fitting for describing the amount of fluctuation of a classical trajectory when we formulate a rigorous Feynman path integral following the idea due to Dirac and Feynman. This fact is illustrated probabilistically in what follows.

The actual expression and computations of the propagator are given successively as follows:

We follow the Lagrangian dynamics. The possible trajectories are sample paths $y(s), s \in[0,1]$, expressed in the form

$$
y(s)=x(s)+\sqrt{\frac{\hbar}{m}} B(s),
$$

where $B(t)$ is an ordinary Brownian motion. Hence the action $S$ is expressed in the form

$$
S=\int_{0}^{t} L(y(s), \dot{y}(s)) d s
$$

Note that the bridged effect is done by putting the delta-function $\delta_{0}\left(y(t)-y_{2}\right)$, where $y_{2}=x(t)$.

We have

Theorem The quantum mechanical propagator $G\left(0, t ; y_{1}, y_{2}\right)$ is given by the following average

$$
G\left(0, t ; y_{1}, y_{2}\right)=\left\langle N \exp \left[\frac{i}{\hbar} \int_{0}^{t} L(y, \dot{y}) d s+\frac{1}{2} \int_{0}^{t} \dot{B}(s)^{2} d s\right] \delta_{o}\left(y(t)-y_{2}\right)\right\rangle
$$

where $N$ is the normalizing constant. 
Actual computations for given potentials (including those have some singularity at the boundary) give us the propagator.

It should be noted that there are generalized white noise functionals in the above expectation. Namely, they are delta functions, in fact the Donsker's delta function $\delta_{o}\left(y(t)-y_{2}\right)$ and Gauss kernels, one of which implicitly appears in the action and the other is $\exp \left[\frac{1}{2} \int_{0}^{t} \dot{B}(s)^{2} d s\right]$ with a multiplicative renormalizing constant. This generalized functional serves for flattening effect of the white noise measure. One may ask why the latter functional is so. An intuitive answer to this question is as follows: If we write a Lebesgue measure (exists only virtually) on $E^{*}$ by $d L$, the white noise measure $\mu$ may be expressed in the form $\exp \left[-\frac{1}{2} \int_{0}^{t} \dot{B}(s)^{2} d s\right] d L$. Hence, the factor in question is put to make the measure $\mu$ to be the flat measure $d L$. In fact, this makes sense eventually.

Returning to the formula (2), it is important to note that the integrand (inside the angular bracket) is integrable, in other words, to see that it is a bilinear form of a generalized functional and a test functional.

In general, the integrand is expressed as a product of a test functional and a functional of the form $\varphi(x) \cdot \delta(\langle x, f\rangle-a), f \in L^{2}(R), a \in R$. To this end, we have to prepare some notes.

The first one is short. Since a sample function $x$ is a genealized function, so the canonical bilinear form $\langle x, \xi\rangle$ is defined for $\xi \in E$ (pointwise in $x$ and $\xi)$. For our purpose it is necessary to extend the bilinear form to $\langle x, f\rangle$, where $f$ is in $L^{2}(R)$. This can be defined as a stochastic bilinear form, although it is no more continuous in $f$.

The next note is important. In general, the formula involves a product of functionals of the form $\varphi(x) \cdot \delta(\langle x, f\rangle-a), f \in L^{2}(R), a \in \mathbf{C}$. To give a correct interpretation to the expectation (2), it should be checked that it can be regarded as a bilinear form of a test functional and a generalized functional. The following assertion answers this question.

Proposition (Streit et al [14]) Let $\varphi(x)$ be a generalized white noise functional. Assume that the $T$-transform $(T \varphi)(\xi), \xi \in E$, of $\varphi$ is extended to a functional on $L^{2}(R)$, in particular function of $\xi+\lambda f$, and that $(T \varphi)(\xi-\lambda f)$ is an integrable function of $\lambda$ for any fixed $\xi$ and $\lambda$. If the Fourier transform of $(T \varphi)(\xi-\lambda f)$ is a $U$-functional, then the pointwise product $\varphi(x) \cdot \delta(\langle x, f\rangle-a)$ is defined and is a generalized white noise functional.

Proof. First a formula for the $\delta$-function is provided.

$$
\delta_{a}(t)=\delta(t-a)=\frac{1}{2 \pi} \int e^{i a \lambda} e^{-i \lambda x} d \lambda
$$


(in distribution sense).

Hence, for $\varphi \in(S)^{*}$ and $f \in L^{2}(R)$ we have

$$
\begin{aligned}
T(\varphi(x) \delta(\langle x, f\rangle-a)) \xi) & =\frac{1}{2 \pi} \int e^{i a \lambda} e^{-i \lambda\langle x, f\rangle} e^{i\langle x, \xi\rangle} \varphi(x) d \mu(x) d \Lambda \\
& =\frac{1}{2 \pi} \int e^{i a \lambda}(T \varphi)\left(\xi^{\lambda} f\right) d \lambda .
\end{aligned}
$$

By assumption this determines a $U$-functional, which means the product $\varphi(x) \cdot \delta(\langle x, f\rangle-a)$ makes sense and it is a generalized white noise functional.

Example A Gauss kernel $\varphi_{c}(x)=N \exp \left[c \int x(t)^{2} d t\right]$.

The following cases are fitting.

i) The case $c$ is real and $c<0$.

ii) The case $c=\frac{1}{2}+i a, a \in R$.

The same expression as in i), and it is shown that Proposition is applied.

Recent developments. Now we should like to mention that there are many successful computations of various propagators. It is easy to see that in the cases i) free particle, ii) simple harmonic oscillator, iii) the Albeverio-HoeghKrohn potential which is the Fourier transform of a measure, the results obtained by our method are in agreement with the known propagators, respectively. In addition, some more interesting cases, including those with much singular potentials and time depending potentials, we have satisfactory results in the recent developments.

Example 1. Kuna, Streit and Westerkampf [14] obtained explicit formulae in the cases:

1) A time depending Lagrangian of the form

$$
L(x(t), \dot{x}(t), t)=\frac{1}{2} m(t) \dot{x}(t)^{2}-k(t)^{2} x(t)^{2}-f(t) x(t),
$$

where $m(t), k(t)$ and $f(t)$ are smooth funtions.

2) A singular potential $V(x)$ of the form

$$
V(x)=\sum_{n} c^{-n^{2}} \delta_{n}(x), \quad c>0,
$$

and others. 
Example 2. C. Bernidos' results [15] on polymer entanglements.

Chern-Simons functional integral See Albeverio-Sengupta [2].

The paper [2] gives us an interesting problem, where white noise analysis is applied. Namely, the authors propose a 3-dimensional gauge theory based on the Chern-Simons action $C S(A)$, where $A$ is a connection over a 3 -manifold that runs through $\mathcal{A}$. Namely, there appears a functional integral of the form

$$
\int_{\mathcal{A}} \exp [i C S(A) \phi(A) D A .
$$

Under various assumptions, $A$ can be expressed in the form

$$
A=a_{0} d x_{0}+a_{1} d x_{1}+a_{2} d x_{2},
$$

where $a_{i}$ is a Lie algebra-valued function on $R^{3}$.

The problem, if we understand correctly, is to define the integral

$$
\frac{1}{N} \int e^{i \kappa /(2 \pi)\left\langle a_{0}, f_{1}\right\rangle} \phi\left(a_{0}, f_{1}\right) D a_{0} D f_{1},
$$

where $f_{1}=\partial_{2} a_{1}$.

Now it is interesting to consider a functional $e^{c\left\langle a_{0}, f_{1}\right\rangle}$. It has some similarity to the Gauss kernel discussed before, but it poses a new problem regarding the inner product of two independent white noises. We note that this problem can be discussed within our framework.

\section{Infinite dimensional rotation group}

This section is devoted to the harmonic analysis that comes from the infinite dimensional rotation group, which is one of our favorite tools in white noise analysis.

Definition A continuous linear homeomorphism $g$ acting on $E$ is called a rotation of $E$ if the following equality holds for every $\xi \in E$;

$$
\|g \xi\|=\|\xi\|
$$

The collection of all rotations forms a group under the usual product, and is denoted by $O(E)$.

Definition Introduce compact-open topology to $O(E)$ to have infinite dimensional rotation group. 
The adjoint transformation $g^{*}$ on $E^{*}$ is defined, and the collection $O^{*}\left(E^{*}\right)$ $=\left\{g^{*} ; g \in O(E)\right\}$ forms a group and isomorphic to $O(E)$. We know that the white noise measure is invariant under $g^{*} \in O^{*}\left(E^{*}\right)$.

We are now ready to discuss harmonic analysis arising from $O^{*}\left(E^{*}\right)$.

Since our infinite dimensional rotation group is quite big, indeed it is neither compact nor locally compact, it seems useful to take two subclasses of significant subgroups and investigate their roles. We are particularly interested in essentially infinite dimensional rotations. There is a criterion for this property.

We define average power a.p. $\left(g_{\pi}\right)$ of a rotation $g_{\pi}$ which comes from a permutation $\pi$ :

$$
\text { a.p. }\left(g_{\pi}\right)(x)=\lim \sup _{N \rightarrow \infty} \frac{1}{N} \sum_{1}^{N}\left\langle x, \xi_{\pi(n)}-\xi_{n}\right\rangle^{2} .
$$

Definition If a.p. $(g)(x)$ is positive $\mu$-a.e., then we call $g_{\pi}$ essentialy infinite dimensional. Contrary to this case, if a.p. $\left(g_{\pi}\right)(x)=0$ almost surely, then $g_{\pi}$ is said to be approximated by finite dimensional rotations.

We can see that there are many members in the Lévy group (see Example below) that are essentially infinite dimensional.

Example Pairwise permutation of the coordinates. The average power is equal to 2 .

It is interesting to note that there should be an intimate connection between the Lévy group and the Lévy Laplacian (e.g. the forthcoming paper by $\mathrm{Si} \mathrm{Si}$ and the author).

\section{The Windmill subgroup.}

There is another subgroup of $O(E)$ that contains essentially infinite dimensional transformation. It is a windmill subgroup $\mathcal{W}$, which is defined in the following manner. Take $E$ to be the Schwartz space $S$ and take a sequence $n(k)$ of positive integers satisfying the condition

$$
(n(k+1)-n(k)) \frac{n(k+1)}{n(k)} \leq K,(K>1) .
$$

Let $\xi_{n}, n \geq 0$, be the complete orthonormal system in $L^{2}(R)$ such that $\xi_{n}$ is the eigenfunction of $A$ as defined before: $A \xi_{n}=2(n+1) \xi_{n}$. Denote by $E_{k}$ the $(n(k+1)-n(k))$-dimensional subspace of $E=S$ that is spanned by $\left\{\xi_{n(k)+1}, \xi_{n(k)+2}, \cdots, \xi_{n(k+1)}\right\}$. Let $G_{k}$ be the rotation group acting on $E_{k}$. Then, $\mathcal{W}=\mathcal{W}(\{n(k)\})$ is defined by 


$$
\mathcal{W}=\otimes_{k} G_{k}
$$

Definition The subgroup $\mathcal{W}$ is called a windmill subgroup.

We can show that $\mathcal{W}$ describes interesting properties of our harmonic analysis.

\section{Infinite dimensional unitary group}

We may assume that a complexification of white noise $\left(E_{c}^{*}, \nu\right)$ is known. Let $z \in E_{c}^{*}$ be of the form:

$$
z=x+i y, \quad x, y \in E .
$$

The complex white noise measure $\nu$ is the product of white noise measures $\mu_{1}$ and $\mu_{2}$ with variance $1 / 2$ :

$$
\nu=\mu_{1} \times \mu_{2} .
$$

Now the unitary group $U\left(E_{c}\right)$ is defined. It is a collection of all transformations $g$ on $E_{c}$ such that

1. $g$ is a linear homeomorphism of $E_{c}$,

2. $g$ preserves the complex $L^{2}(R)$-norm:

$$
\|g \eta\|=\|\eta\|, \quad \zeta \in E_{c} .
$$

Definition The topological group $U\left(E_{c}\right)$ is called the infinite dimensional unitary group.

The adjoint $g^{*}$ of $g$ in $U\left(E_{c}\right)$ is defined and we see that

$$
g^{*} \nu=\nu .
$$

Hence, we are given a unitary operator $U_{g}$ defined by

$$
U_{g} \varphi(z)=\varphi\left(g^{*} z\right), \varphi \in\left(L_{c}^{2}\right) .
$$

Under the usual product the collection of $U_{g}$ 's forms a topological group that is isomorphic to the group $U\left(E_{c}\right)$.

It is noted that the infinite dimensional rotation group $O(E)$ may be identified with a subgroup of $U\left(E_{c}\right)$. 
Subgroups of $U\left(E_{c}\right)$.

Many results below are known, but reviews or rephrasements are useful.

1) Conformal group.

In the $R^{d}$-parameter case, if the basic nuclear space is taken to be $D_{0, c}$, then we are given the conformal group $C(d)$ which is a subgroup of $O(E)$ as was briefly mentioned before. Hence, the complex form of $C(d)$, denote it by $C_{c}\left(D_{0}\right)$, acting on the space $D_{0, c}$, is a subgroup of $U\left(D_{0, c}\right)$. We call it a complex conformal group. It is locally isomorphic to the (real) linear group $S O(d+1,1)$ and is generated by one-parameter groups including whiskers as many as $\frac{(d+1)(d+2)}{2}$. Their generators are as follows:

$$
\begin{aligned}
s & =-\frac{d}{d u_{i}}, \quad i=1,2, \ldots, d, \\
\tau & =r \frac{d}{d r}+\frac{d}{2}, \quad r=|u|, \\
r_{j, k} & =u_{j} \frac{d}{d u_{k}}-u_{k} \frac{d}{d u_{j}}, 1 \leq j \neq k \leq d, \\
\kappa_{j} & =u_{j}^{2} \frac{d}{d u_{j}}+u_{j}, j=1,2, \ldots, d .
\end{aligned}
$$

They correspond to the shifts, isotopic dilation, rotations on $R^{d}$ and special conformal transformations, respectively.

2) Heisenberg group.

From now on, one can see the effective use of complex white noise. Now take $E_{c}$ to be $\mathcal{S}_{c}=\mathcal{S}+i \mathcal{S}, \mathcal{S}$ being the Schwartz space.

2.1) The gauge transformation $I_{t}$ is defined by

$$
I_{t}: \zeta(u) \longrightarrow I_{t} \zeta(u)=e^{i t} \zeta(u) .
$$

Obviously $I_{t}$ is a member of $U\left(E_{c}\right)$, and $\left\{I_{t}\right\}$ forms a continuous one parameter subgroup, periodic with period $2 \pi$. Let $I$ be the identity.

$$
\begin{aligned}
I_{t} I_{s} & =I_{t+s}, \quad t, s \in R, \\
I_{t+2 \pi} & =I_{t}, \\
I_{t} & \rightarrow I \text { as } t \rightarrow 0 .
\end{aligned}
$$

The group $\left\{I_{t}, t \in R\right\}$ is called the gauge group. Let $U_{t}$ be defined by $U_{I_{t}}$. This unitary group has only point spectrum on the subspace $H_{n}$. The eigenspace belonging to the eigenvalue $-n+2 k$ is $H_{(n-k, k)}$. Hence, the space $H_{n}, n>1$, is 
classified by $I_{t}$ into its subspaces $H_{(n-k, k)}$. The generator of the gauge group is $i I$.

Remark. The operator $I_{t}$ extends to a more general gauge transformation, where $i t$ is replaced by itf, $f \in E$, so that we have a collection of generators that span a space isomorphic to a nuclear space $E$, hence a nuclear Lie algebra is given.

2.2) The shifts $S_{t}^{j}$ with generators

$$
-\frac{\partial}{\partial u_{j}}, \quad j=1,2, \cdots, d
$$

2.3) Multiplication $\pi_{t}^{j}, j=1,2, \cdots, d$. Let them be defined to be the conjugate to the shifts via the Fourier transform $\mathcal{F}$ :

$$
\pi_{t}^{j}=\mathcal{F} S_{t}^{j} \mathcal{F}^{-1} .
$$

Definition The subgroup generated by the gauge group, the shifts and the multiplication is called the Heisenberg group.

4) The Fourier-Mehler transforms $\mathcal{F}_{\theta}(d=1)$.

It is possible to consider the fractional power of the ordinary Fourier transform. It is defined by the integral kernel $K_{\theta}(u, v)$ :

$$
K_{\theta}(u, v)=(\pi(1-\exp [2 i \theta]))^{-1 / 2} \exp \left[-\frac{i\left(u^{2}+v^{2}\right)}{2 \tan \theta}+\frac{i u v}{\sin \theta}\right] .
$$

It defines an operator $\mathcal{F}_{\theta}$ by writing

$$
\left(\mathcal{F}_{\theta} \zeta\right)(u)=\int_{-\infty}^{\infty} K_{\theta}(u, v) \zeta(v) d v
$$

where $\theta \neq \frac{1}{2} k \pi, k \in Z$. Particular choices of $\theta$ give

$$
\mathcal{F}_{\pi / 2}=\mathcal{F}, \quad \mathcal{F}_{(3 / 2) \pi}=\mathcal{F}^{-1} .
$$

Thus, we have obtained a periodic one-parameter unitary group including the Fourier transform and its inverse.

The infinitesimal generator of $\mathcal{F}_{\theta}$ is denoted by if and is expressed in the form

$$
\text { if }=-\frac{1}{2} i\left(\frac{d^{2}}{d u^{2}}-u^{2}+I\right) .
$$


Observing the commutation relations among the generators, so as to have a finite dimensional Lie algebra, either real or complex form, we are given a generator $\sigma^{\prime}$ of the form

$$
\sigma^{\prime}=\frac{1}{2}\left(\frac{d^{2}}{d u^{2}}+u^{2}\right) .
$$

We are now interested in probabilistic roles or meanings of this operator in quantum dynamics (as the repulsive oscillator).

It is convenient to take $\sigma=\sigma^{\prime}+\frac{i}{2} I$, namely we have

$$
\sigma=\frac{1}{2}\left(\frac{d^{2}}{d u^{2}}+u^{2}+i I\right)
$$

A one-parameter group with the generator $\sigma$ can be defined locally in spacetime. See e.g. [16].

\section{Lie algebras of infinitesimal generators.}

We have so far various infinitesimal generators. For simplicity we consider the case $d=1$, that is the case of one-dimensional parameter complex white noise.

The algebraic structure of the space spanned by the generators is helpful for applications to quantum dynamics and differential geometry.

We list the generators so far obtained (for the case $d=1$ ).

$$
I, s, \tau, \kappa, \pi, f, \sigma
$$

Proposition Based on the set of operators

$$
\{i I, s, i \pi, \tau, f, \sigma\}
$$

we have 6-dimensional complex Lie algebra $\mathbf{g}$.

Proposition The abgebra generated by $\{i I, s, i \pi\}$ is a radical of $\mathbf{g}$.

In the multi-dimensional, say $d$-dimensional, case the rotations $r_{j, k}, 1 \leq$ $j, k \leq d$, are involved in the algebra for conformal group:

$$
r_{j, k}=u_{j} \frac{\partial}{\partial u_{k}}-u_{k} \frac{\partial}{\partial u_{j}}, \quad 1 \leq j \neq k \leq d .
$$

The algebraic structure of the Lie algebra involving the $r_{j, k}$ does not make much difference from the case $d=1$. 
Now it seems necessary to give an interpretation to the fact that the generator $\kappa$ of the special conformal transformation is a particular one, being excluded from $\mathbf{g}$.

1) The reason why the $\kappa$ has been taken.

i) It is a good candidate to be introduced among the possible expressions of generators expressed in the the form $a(u) \frac{d}{d u}+\frac{1}{2} a^{\prime}(u)$. If the basic nuclear space $E$ is taken to be $D_{0}$, the $\kappa$ is acceptable with $a(u)=u^{2}$. As a result, we have proved that the algebra generated by those possible generators is isomorphic to $\operatorname{sl}(2, R)$.

ii) Similar to $s$, the $\kappa$ is transversal to $\tau$, which defines a flow of the Ornstein-Uhlenbeck process (flow).

2) On the other hand, there are crucial reasons why $\kappa$ should not be involved in the algebra $\mathbf{g}$.

i) In order to introduce the $\kappa$ we need particular space like $D_{0}$, instead of a familiar space $S_{c}$.

ii) It does not satisfy favorable commutation relations with other favorable ones.

\section{References}

1. L. Accardi et al, Selected papers of Takeyuki Hida. World Scientific Pub. Co. 2001.

2. S. Albeverio and A. Sengupta, The Chern-Simons functional integral as an infinite dimensional distribution. Nonlinear Analysis, Theory, Methods and Applications. 30 (1997), 329-335.

3. T. Hida, Analysis of Brownian functionals. Carleton Math. Notes no.13, 1975.

4. T, Hida Brownian motion. Springer-Verlag, 1980.

5. K. Itô, Multiple Wiener integrals. J. Math. Soc. Japan. 3 (1951) , 157-169.

6. T. Hida, H.-H. Kuo, J. Potthoff and L. Streit, White noise. An Infinite dimensional calculus. Kluwer Academic Pub. 1993.

7. Hida and $\mathrm{Si} \mathrm{Si}$, Innovation approach to random fields: An application of white noise theory. World Sci. Pub. Co. 2004.

8. T. Hida and Si Si, Lectures on white noise functionals. Monograph tp appear in World Sci. Pub. Co.

9. P. Lévy, Processu stochastiques et mouvement brounien. Gauthier-Villars, 1848.

10. P. Lévy, Problèmes concrets d'analyse fonctionelle. Gauthier-Villars, 1951.

11. P. Lévy, Random functions: General theory with special reference to Lapalcian random functions. Univ. of Calif. Pub. in Statistics. I, 12 (1953), 331-388.

12. Si Si, Effective determination of Poisson noise. IDAQP 6 (2003), 609-617.

13. L. Streit and T. Hida, Generalized Brownian functional and Feynman integral. Stochastic Processes and Appl. 16 (1983), 55-69.

14. T. Kuna, L. Streit and W. Westerkampf, Feynman integrals for a class of exponentially growing potentials. J. Math. Phys. 39 (1998), 4476-4491. 
15. C. C. Bernido and M.V. Carpio-Bernido, White noise functional approach to polymer entanglements. Proc. Stochastic Analysis: Classical and Quantum. World Sci. Pub. Co. (2005), 1 - 12.

16. P. Topping, Repulsion and quantization in almost harmonic maps, and asymptotics of the harmonic map flow. Ann. Math. 159 (2004), 465-534. 